Michael W. M. Roos*

\title{
Die Komplexitätsökonomik und ihre Implikationen für die Wirtschaftspolitik
}

DOI 10.1515/pwp-2015-0024

Zusammenfassung: Dieser Aufsatz stellt die Komplexitätsökonomik als Alternative zur neoklassischen Ökonomik vor und zeigt, wie sich die Sicht der Komplexitätsökonomik auf die Makroökonomie von derjenigen des neoklassischen Mainstreams unterscheidet. Nach einer theoretischen Einführung wird mikro- und makroökonomische Evidenz vorgestellt, die die Interpretation der Gesamtwirtschaft als komplexes adaptives System unterstützt. Komplexe adaptive Systeme weisen Pfad- und Kontextabhängigkeit auf, sind nur eingeschränkt prognostizierbar und nicht zentral zu steuern. Anschließend wird diskutiert, welche Implikationen die Komplexitätsökonomik für die Wirtschaftspolitik hat. Eine zentrale Aussage ist, dass es in komplexen adaptiven Systemen kaum möglich ist, optimale Politiken $\mathrm{zu}$ bestimmen und die Ökonomie durch zentrale wirtschaftspolitische Maßnahmen in einer gewünschten Weise zu lenken.

Schlüsselwörter: ökonomische Methoden, computergestützte Verfahren, Simulationsmodelle, makroökonomische Politikmaßnahmen, makroökonomische Aspekte der Finanzwissenschaft, allgemeine Prognosen

JEL: B4, C63, E6

\section{Einleitung}

Die globale Finanzkrise von 2007-2009 und ihre noch immer andauernden Folgen stellen die Volkswirtschaftslehre, insbesondere die Makroökonomik, vor große Herausforderungen. Einerseits muss die Makroökonomik erklären, wie es zur größten Wirtschaftskrise seit den dreißiger Jahren kommen konnte, obwohl Robert Lucas (2003) und mit ihm viele andere Makroökonomen geglaubt hatten, dass das Problem der Konjunkturschwankungen und der Depressionsvermeidung gelöst wäre. Andererseits wird erwartet, dass die Makroökonomik Antworten auf die
Frage gibt, wie mit den Folgen der Krise politisch umgegangen werden soll und wie zukünftige Krisen dieser Art verhindert werden können.

Während die Makroökonomik in Politik und Gesellschaft viel an Glaubwürdigkeit und Kredit verloren hat (z. B. Buiter 2009, The Economist 2009, Trichet 2012), sieht die Mehrheit der Ökonomen das Fach als Ganzes nicht in einer Krise, sondern arbeitet mit Hochdruck an einer Erweiterung und Modifikation der vorhandenen Modelle innerhalb des bestehenden Paradigmas.

In diesem Aufsatz möchte ich darstellen, dass es in der Volkswirtschaftslehre nicht nur mehr derselben traditionellen neoklassischen Theorien ${ }^{1}$ gibt, sondern auch alternative Ansätze, die zu anderen Einsichten führen. Dabei möchte ich vor allem herausarbeiten, wie sich die Komplexitätsökonomik als eine der sogenannten heterodoxen Theorien von der Mainstream-Makroökonomik unterscheidet und zeigen, dass sie gravierende Implikationen für die Wirtschaftspolitik und auch für die wirtschaftspolitische Beratung durch Ökonomen hat. Heterodoxe Ökonomik ist ein Sammelbegriff für eine Vielzahl verschiedener Ansätze, die zum Teil auf eine recht lange Vergangenheit zurückblicken. Zu nennen wären zum Beispiel die Evolutorische Ökonomik, die Postkeynesianische Ökonomik oder die Österreichische Ökonomik. ${ }^{2}$ In ihrer jeweiligen Form und Schwerpunktsetzung unterscheiden sich diese und andere heterodoxe Ansätze. Was sie vereint, ist ihre kritische bis ablehnende Haltung gegenüber dem neoklassischen Mainstream und seinen Kernannahmen. Im Zentrum der Kritik stehen dabei vor allem das Verhaltensmodell des homo oeconomicus und die Bedeutung des Gleichgewichtskonzepts. Mit Ausnahme der Verhaltensökonomik, die man mittlerweile zum ökonomischen Mainstream zählen kann, spielen die meisten dieser Ansätze in der akademischen Lehre und Forschung praktisch keine Rolle und werden von der Mehrheit der akademischen Ökonomen sehr kritisch gesehen. Jedoch wünschen sich zunehmend mehr Studierende eine Auseinandersetzung mit diesen heterodoxen Theorien und mehr Pluralismus in Forschung und

1 Neoklassisch ist hier im methodischen Sinn gemeint.

2 Siehe Dequech (2007) und Lawson (2006) für Übersichten.
*Kontaktperson: Michael W. M. Roos, Ruhr-Universität Bochum Fakultät für Wirtschaftwissenschaft, LS Makroökonomik, Universitätsstrasse 150, 44801 Bochum, E-Mail:michael.roos@ruhr-uni-bochum.de 
Lehre (siehe Netzwerk Plurale Ökonomik ${ }^{3}$ ). Auch viele heterodoxe Ökonomen kritisieren die dogmatische Haltung des Mainstreams und sehen in seiner Intoleranz gegenüber einer Vielfalt von Forschungsansätzen und Modellen ein großes Problem für die Disziplin (Heise 2015).

Ein wichtiger Grund für die Skepsis vieler Ökonomen gegenüber heterodoxen Theorien ist, dass diese sich häufig scheinbar auf eine bloße Kritik der Neoklassik beschränken und aus Sicht des Mainstreams nicht zeigen, wie sie in konkreten Fragen zu Ergebnissen kommen. Die häufige Ablehnung mathematischer Modellierung und ökonometrischer Analysen führt dazu, dass Mainstreamökonomen heterodoxe Ansätze als wenig wissenschaftlich ansehen. ${ }^{4}$ Wie bereits erwähnt, stellt die Verhaltensökonomik hier eine Ausnahme dar. Nicht zuletzt aufgrund zahlreicher bedeutungsvoller Erkenntnisse ist ihre wichtige Methode der ökonomischen Experimente in der Wissenschaft mittlerweile ebenso anerkannt wie die Versuche alternativer formaler Modellbildung unter Einbezug verhaltensökonomischer Konzepte (vgl. Croson und Gächter 2010). Auch war es für ihre Akzeptanz sicher hilfreich, dass viele Verhaltensökonomen die neoklassische Methodologie nicht grundsätzlich ablehnen.

Ein Ansatz, der Elemente aus vielen Richtungen der heterodoxen Ökonomik enthält, ist die sogenannte Komplexitätsökonomik (complexity economics). ${ }^{5}$ Diese Forschungsrichtung, basierend auf der Komplexitätstheorie (vgl. Miller und Page 2007, Wolfram 2002) bzw. Systemtheorie (von Bertalanffy 1976), versteht die Ökonomie als komplexes adaptives System. $\mathrm{Zu}$ ihren Analysewerkzeugen zählen die Netzwerktheorie, die Chaostheorie und die Mathematik nichtlinearer Systeme sowie die agentenbasierte Modellierung. Im Gegensatz zu vielen anderen heterodoxen Forschungsrichtungen verfügt die Komplexitätsökonomik also über ein umfangreiches Repertoire an etablierten formalen Methoden. Die Komplexitätstheorie wird mit Erfolg bereits in anderen Wissenschaften wie der Epidemiologie, der Ökologie, der Physik, der Neurowissenschaft oder der Meteorologie verwendet. In der Politikwissenschaft gibt es mit dem Historischen Institutionalismus eine Forschungsrichtung, die ebenfalls auf der Komplexitätstheorie beruht. Ma (2007) sieht darin das Po-

3 https://www.plurale-oekonomik.de/.

4 Allerdings muss auch gesagt werden, dass der Mainstream auch mathematische heterodoxe Ansätze wie von Richard Goodwin, Luigi Pasinetti, Steve Keen, Duncan Foley und anderen häufig ablehnt. Ich danke einem der Gutachter für den Hinweis.

5 Vgl. Arthur (2015), Beinhocker (2007), Colander (2000), Colander et al. (2011), Colander und Kupers (2014), Durlauf (1998), Elsner et al. (2014), Fontana (2008), Kirman (2011). tential für einen Paradigmenwechsel in der Politikwissenschaft und sieht enge Parallelen zur ähnlich gelagerten Diskussion in der Volkswirtschaftslehre.

Ich werde in diesem Essay zeigen, dass die Komplexitätsökonomik eine radikal andere Sicht auf die Ökonomie hat als die Standardmakroökonomik und in vielen Fällen zu anderen Aussagen bzw. Prognosen kommt. Es ist nicht das Ziel dieses Aufsatzes, eine Zusammenfassung einzelner makroökonomischer Modelle aus der Komplexitätsökonomik zu liefern. ${ }^{6}$ Vielmehr soll die zentrale Botschaft vermittelt werden, dass die Komplexitätsökonomik einen von den neoklassischen Methoden grundverschiedenen Ansatz darstellt, der mit anderen Methoden arbeitet, auf anderen Prämissen beruht und andere Fragen stellt. ${ }^{7}$ Anschließend wird Evidenz diskutiert, die mit Aussagen der Komplexitätsökonomik im Einklang steht und die Auffassung von der Ökonomie als komplexes adaptives System unterstützt. Schließlich werden einige Überlegungen angestellt, welche Implikationen die Komplexitätsökonomik für die Wirtschaftspolitik und die wirtschaftspolitische Beratung hat.

Die Komplexitätsökonomik hat eine sehr aktive Scientific Community, die ihre Arbeiten in einer ganzen Reihe von angesehenen internationalen Journalen veröffentlicht, z. B. Physica A, Physica D, Chaos, Complexity, Advances in Complex Systems, und die über ein erhebliches gedankliches und methodisches Repertoire verfügt. Allerdings sind die Community und ihre Arbeiten dem ökonomischen Mainstream weitgehend unbekannt. Neben der Darstellung relevanter Politikimplikationen soll dieser Essay auch einen Anstoß zur Diskussion und zum Überdenken traditioneller Positionen in Forschung, Lehre und Wirtschaftspolitik liefern.

\section{Standardmakroökonomik}

Bevor ich die wesentlichen Merkmale der Komplexitätsökonomik darstelle, möchte ich komprimiert die wichtigsten Charakteristika der Standardmakroökonomik zusam-

6 Beispiele für solche Modelle sind Assenza und Delli Gatti (2013), Deissenberg et al. (2008), Delli Gatti et al. (2011), Dosi et al. (2010), Dosi et al. (2013), Mandel et al. (2009), Salle et al. (2013).

7 Man kann Arthur (2015) folgend die neoklassische Ökonomik als einen Spezialfall der Komplexitätsökonomik sehen. Demnach betrachtet die Komplexitätsökonomik das Gleichgewicht als einen Sonderfall und die Abwesenheit des Gleichgewichts als den Normalzustand einer Ökonomie. Aus diesem Grund ist dann die neoklassische Gleichgewichtsanalyse ein Spezialfall der wesentlich allgemeineren Komplexitätsökonomik, die keine Gleichgewichte voraussetzt. 
menfassen, um die Unterschiede besser herausarbeiten $\mathrm{zu}$ können.

Der gegenwärtige Goldstandard in der makroökonomischen Forschung sind die DSGE-Modelle (dynamic stochastic general equilibrium models). Dies sind mikrofundierte allgemeine Gleichgewichtsmodelle mit einer schwach ausgeprägten endogenen Dynamik. Die Dynamik der Variablen stammt aus exogenen Zufallsgrößen. Wenn ein zufälliger Schock auftritt, passen die Haushalte und Unternehmen ihre Entscheidungsvariablen wie Konsum oder Arbeitsnachfrage in nutzen- bzw. gewinnmaximierender Weise und mit rationalen Erwartungen an die veränderte Situation an und lösen damit weitere Änderungen auf den Märkten aus. Wenn keine neuen Schocks auftreten, führen die optimalen Anpassungen auf den einzelnen Märkten früher oder später dazu, dass wieder ein allgemeines Gleichwicht hergestellt wird, in dem sich die makroökonomischen Variablen nicht mehr ändern bzw. mit konstanten Änderungsraten wachsen (Steady State). Da viele einmalige Schocks nur zu sehr kurzfristigen Reaktionen der makroökonomischen Variablen führen würden, was im Widerspruch zur empirischen Evidenz steht, wird zumeist angenommen, dass die Schocks autokorreliert sind, wodurch die Konvergenz zum Gleichwicht zeitlich gestreckt wird.

In dieser Literatur wird sehr viel Wert auf eine Mikrofundierung gelegt. Das bedeutet, dass alle Aggregatvariablen aus den rationalen, axiomatisch fundierten Entscheidungen von Individuen - Haushalten und Unternehmen hergeleitet werden müssen. Um das Aggregationsproblem bewältigen zu können, wird angenommen, dass die Individuen identisch sind und daher durch einen repräsentativen Agenten dargestellt werden können, wodurch die Makroebene mit der Mikroebene zusammenfällt. Selbst wenn eine begrenzte Art von Heterogenität zugelassen wird, z. B. durch die Annahme, dass jeder Haushalt eine besondere Art von Arbeit anbietet, wird diese durch weitere Hilfsannahmen wie vollkommene Versicherungsmärkte bzw. contingent claims an anderer Stelle wieder aufgehoben. ${ }^{8}$ Die auftretenden Schocks sind daher auch Aggregatschocks, die alle Individuen eines Sektors in derselben Weise betreffen.

Die Individuen sind rationale intertemporale Nutzenbzw. Gewinnmaximierer mit stabilen Präferenzen und konstanten Technologien, so dass ihr Verhalten durch un-

8 Dies ist z. B. im bekannten Modell von Smets and Wouters (2003) der Fall. Eine wachsende makroökonomische Literatur verbindet heterogene Agenten mit unvollständigen Märkten, vgl. Heathcote et al. (2009), Krusell und Smith (2006) für Übersichten. Jedoch ist auch in dieser Literatur die Behandlung von Heterogenität sehr eingeschränkt, z. B. durch die Konzentration auf idiosynkratische Schocks. veränderliche Funktionen wie die Konsum-Euler-Gleichung oder Preissetzungsfunktionen beschrieben wird. Das konkrete Verhalten kann durch Schocks in Präferenzen, der Technologie oder in Preissetzungsparametern beeinflusst werden, aber die Verhaltensmuster sind unveränderlich.

Üblicherweise sind die DSGE-Modelle linear bzw. linearisiert in der Nähe des Steady States. Daraus folgt eine Proportionalität zwischen der Stärke des Schocks und der Größe der Effekte auf die makroökonomischen Variablen.

Raum wird gewöhnlich nicht modelliert und Zeit ist abstrakt. Zwar sind die DSGE-Modelle in der Regel mit empirischen Daten kalibriert, so dass eine Zeiteinheit normalerweise einem Quartal entspricht. Jedoch ist die Zeit unhistorisch in dem Sinne, dass es für die Antwort des Systems auf einen Impuls keine Rolle spielt, zu welchem Zeitpunkt der Schock auftritt. Die resultierende Anpassung zum Gleichgewicht verläuft immer gleich. ${ }^{9}$

\section{Komplexitätstheorie}

Die Komplexitätsökonomik versteht die Wirtschaft als komplexes adaptives System und wendet Einsichten und Methoden der Komplexitätswissenschaft (complexity science) oder Komplexitätstheorie in der Wirtschaftswissenschaft an. Die Komplexitätstheorie beschäftigt sich disziplinübergreifend mit der Erforschung komplexer (adaptiver) Systeme, sucht nach gemeinsamen Prinzipien solcher Systeme und stellt geeignete Forschungsmethoden bereit. Ihre Ursprünge hat sie unter anderem in der Kybernetik (Wiener 1948), der Künstlichen-Intelligenz-Forschung (vgl. Shapiro 1992), der Chaostheorie (vgl. Lorenz 1963, May 1976) und der soziologischen Systemtheorie (Luhmann 1984).

Es gibt verschiedene Definitionen komplexer adaptiver Systeme. Eine sehr bekannte Definition ist die von John Holland ${ }^{10}$ :

A Complex Adaptive System (CAS) is a dynamic network of many agents (which may represent cells, species, individuals, firms, nations) acting in parallel, constantly acting and reacting to what the other agents are doing. The control of a CAS tends to be highly dispersed and decentralized. If there is to be any coherent behavior in the system, it has to arise from competition and cooperation among the agents themselves. The overall behavior

9 Mit Unhistorizität ist hier gemeint, dass keine Pfadabhängigkeit vorliegt. Dies ist in Modellen der Komplexitätsökonomik oft anders, was im folgenden Abschnitt ausgeführt wird.

10 John Holland zitiert nach M. Mitchell Waldrop (1992). 
of the system is the result of a huge number of decisions made every moment by many individual agents.

Etwas prägnanter definiert Holland (2006, S. 1): „CAS are systems that have a large numbers of components, often called agents, that interact and adapt or learn.“

Eine wichtige Methode, um komplexe adaptive Systeme abzubilden und zu analysieren, ist die agentenbasierte Modellierung. Mit agentenbasierten Modellen (ABM) können künstliche Gesellschaften, die aus sogenannten Agenten bestehen, geschaffen und mit Hilfe von Computerprogrammen simuliert werden. Durch die Betrachtung von Agenten sind diese Modelle inhärent mikrofundiert. Alle Phänomene auf der Makroebene resultieren in diesen Modellen jedoch nicht nur aus dem individuellen Verhalten der Agenten, wie beispielsweise in den angesprochenen DSGE-Modellen, sondern insbesondere aus deren Interaktion. Im Vergleich zu DSGE- und anderen mathematischen Modellen in der Volkswirtschaftslehre weisen ABM einige Besonderheiten auf. Erstens erlauben sie vielfältige Heterogenität der Agenten, z. B. mit Bezug auf Wissen, Präferenzen, Fähigkeiten, Ressourcen, Verhaltensweisen usw. Zweitens sind die Agenten normalerweise nur begrenzt rational ${ }^{11}$ und keine perfekten Nutzen- oder Gewinnmaximierer. Drittens findet direkte Interaktion zwischen Agenten statt. Diese Interaktion ist normalerweise lokal, d. h. Agenten interagieren nur mit anderen in einer irgendwie definierten Nachbarschaft oder in einem Netzwerk. Begrenzte Rationalität und lokale Interaktion implizieren, dass die Agenten nur partielles Wissen über ihre Umwelt besitzen. Viertens ermöglicht diese direkte Interaktion, dass die Agenten voneinander lernen, Informationen austauschen, Verhaltensweisen imitieren etc. Schließlich handeln alle Agenten jederzeit völlig autonom. Das bedeutet keineswegs, dass ein Agent nicht auf das Verhalten anderer oder Aggregatphänomene reagiert - im Gegenteil. Autonomie heißt, dass das Verhalten nicht über Gleichge-

11 Einer der Gutachter schlug statt „begrenzt rational“ den Begriff „beliebig rational“ vor, um auszudrücken, dass der Grad an Rationalität in ABM nahezu beliebig variiert werden kann. So ist es sowohl möglich, sogenannte „zero intelligence agents“ mit sehr einfachen Verhaltensregeln zu modellieren, als auch Agenten, die anspruchsvolle Optimierungsalgorithmen ausführen und auf komplizierte Art lernen. Für ökonomische Anwendungen sind Modelle mit planenden, antizipierenden und deliberativen Agenten sicher relevanter als „zero intelligence agents“. Allerdings wird in ABM zumeist berücksichtigt, dass zum einen die Entscheidungsprobleme in komplexen Systemen in der Regel nicht wohldefiniert und zum anderen die kognitiven Kapazitäten der Entscheider begrenzt sind. In Abgrenzung zum Rationalitätsbegriff neoklassischer Gleichgewichtsmodelle wird die resultierende Rationalität meist als „,begrenzt“ (bounded) bezeichnet. wichtsbedingungen auf Märkten oder in Interaktionsbeziehungen bestimmt wird. Stattdessen haben Agenten ein Verhaltensrepertoire, dessen sie sich bedienen und das bestimmt, wie sich ein Agent in einer bestimmten Situation verhält. Die Abwesenheit von Gleichgewichtsbedingungen in ABM ist der zentrale Unterschied zu den Modellen der Neoklassik.

Aus Sicht der Komplexitätsökonomik haben Ökonomien als komplexe adaptive Systeme völlig andere Eigenschaften als aus Sicht der neoklassischen Ökonomik. Dies liegt daran, dass sie in der Regel nicht-lineare Dynamiken haben und nicht angemessen durch lineare Modelle beschrieben werden können. Nicht-lineare dynamische Systeme können chaotisch sein oder sich am Rande des Chaos befinden. Obwohl sie deterministisch sind, sind sie dann prinzipiell unvorhersagbar (vgl. Velupillai 2005) und weisen eine extreme Sensitivität bezüglich ihrer Anfangsbedingungen auf. Nicht-lineare Systeme sind auch häufig durch Selbstverstärkungsprozesse, die durch positives oder negatives Feedback ausgelöst werden, gekennzeichnet. Solche Feedbackprozesse können dazu führen, dass kleine exogene Störungen sehr große Effekte auf das System haben, jedoch auch, dass große Schocks vom System neutralisiert werden und sich nur wenig auf Aggregatgrößen auswirken. Komplexe Systeme können Attraktoren haben, die eine Teilmenge des Zustandsraums sind, auf die sich das System zubewegt. Attraktoren sind abgeschlossene Strukturen und können in verschiedenen Formen auftreten, z. B. als einzelner Punkt (stabiles Gleichgewicht) oder als Grenzzyklus, in dem die gleichen Zustände periodisch durchlaufen werden. Ein weiteres Merkmal von komplexen nicht-linearen Systemen ist, dass sie ihre Eigenschaften beim Überschreiten sogenannter kritischer Werte oder tipping points sehr plötzlich ändern können. In der Thermodynamik spricht man von Phasenübergängen, z. B. zwischen dem festen und dem flüssigen Aggregatzustand. Solche Bifurkationspunkte sind kritische Werte eines Systemparameters, an denen sich die Gleichgewichtsstruktur des Systems ändert. So kann sich an einem Bifurkationspunkt die Anzahl oder auch die Stabilität der Gleichgewichte ändern. Schließlich können komplexe nicht-lineare Systeme durch Pfadabhängigkeit und Irreversibilität charakterisiert sein. Pfadabhängigkeit bedeutet, dass das Verhalten eines Systems nicht nur vom aktuellen Zustand, sondern auch von der jeweiligen Vorgeschichte abhängig ist. Ein Prozess ist irreversibel, wenn der Ausgangszustand des Systems nicht wieder hergestellt werden kann. Beispiele dafür sind das Aussterben von Arten oder der $\mathrm{Zu}$ sammenbruch von Klimaprozessen. Pfadabhängige Prozesse können durch geringfügige stochastische Störungen von nahe beieinanderliegen Zuständen zu verschiedenen 
Attraktoren konvergieren. Pfadabhängigkeit und Irreversibilität sind eng verbunden mit dem Konzept der NichtErgodizität. Ein System ist ergodisch, wenn die Zeitverteilung seiner Makrozustände der Ensembleverteilung entspricht. Wenn dies der Fall ist, nimmt das System jeden möglichen Zustand irgendwann auch an. Bei nicht-ergodischen Prozessen wird nicht jeder Zustand erreicht und der langfristig erreichte Zustand hängt vom zufällig bestimmten Pfad ab.

Zusammengefasst ergibt sich ein ganz entscheidender Unterschied zwischen der Weltsicht der neoklassischen Ökonomik und der Komplexitätsökonomik. Im Gegensatz zur Komplexitätsökonomik beschreibt die Neoklassik die Ökonomie durch eindeutige, stabile Gleichgewichte ${ }^{12}$. Das neoklassische System ist normalerweise im Gleichgewicht (oder in der Nähe) und wird nur durch externe Schocks gestört. Nach einem solchen Schock strebt es wieder dem ursprünglichen Gleichgewicht zu. Bei identischen Schocks ist der Anpassungsprozess jedes Mal gleich, so dass sich diese Anpassungen gewissermaßen in abstrakter Zeit abspielen und auch keine Pfadabhängigkeit besteht. Wenn sich exogene Parameter oder Variablen des Systems ändern, z. B. durch wirtschaftspolitische Maßnahmen, ändert sich gewöhnlich auch das Gleichgewicht. Da üblicherweise Modelle mit eindeutigen Gleichgewichten betrachtet werden, können die Auswirkungen politischer Maßnahmen prognostiziert werden, wenn man das neue Gleichgewicht und den Anpassungspfad zwischen den Gleichgewichten ermitteln kann. Wie zuvor beschrieben, sind stabile Gleichgewichte nur ein Sonderfall von Attraktoren in dynamischen Systemen. Von solchen Attraktoren kann es mehrere geben und sie können seltsam (chaotisch) sein wie der bekannte Lorenz-Attraktor (Lorenz 1963). Dann hängt die Dynamik des Systems von den Anfangsbedingungen ab und ist nicht vorhersagbar, obwohl das System deterministisch ist. Durch Parameteränderungen kann es dazu kommen, dass sich die Anzahl der Attraktoren oder ihre Eigenschaften verändern. Es können Bifurkationen auftreten, wodurch das System in qualitativ andere $\mathrm{Zu}$ stände übergeht. Ökonomische Arbeiten, in denen solche seltsamen Attraktoren auftreten, sind z.B. das kaldorianische Multisektor-Konjunkturmodell in Lorenz (1987) oder die mathematische Formalisierung von Minskys Hypothese der finanziellen Instabilität in Keen (1995).

Komplexe adaptive Systeme zeichnen sich durch Selbstorganisationsprozesse aus, durch die ein System

12 Es gibt natürlich neoklassische Modelle mit multiplen Gleichgewichten, dies ist jedoch eher unerwünscht oder wird als Anomalie betrachtet. Häufig werden auch Annahmen getroffen, um multiple Gleichgewichte auszuschließen. ohne zentrale oder äußere Steuerung zu einer stabilen Struktur gelangt, was man als Bewegung hin zu einem Attraktor verstehen kann. Die Selbstorganisation entsteht durch das Zusammenwirken der Agenten und ihre wechselseitige Anpassung aneinander. Ein verwandter Begriff ist die Emergenz, womit ebenfalls die spontane Herausbildung von Systemstrukturen durch die Interaktion der Systemelemente gemeint ist. Das Besondere an emergenten Systemeigenschaften ist, dass sie sich nicht einfach aus den Eigenschaften der Systemelemente bzw. der Agenten ableiten oder vorhersagen lassen, da sie eben erst aus der Interaktion entstehen. Eine Analyse des Gesamtsystems durch das Studium seiner Elemente bzw. kleinerer Subsysteme (Reduktionismus) ist dann nicht geeignet, die Systemeigenschaften zu erfassen, da durch eine isolierte Betrachtung der Elemente deren Interaktion verlorengeht.

Neben der Unvorhersagbarkeit haben diese Eigenschaften komplexer Systeme auch Implikationen für die Steuerbarkeit durch externe Eingriffe wie wirtschaftspolitische Maßnahmen. Wenn sich ein System im Einzugsbereich eines Attraktors (basin of attraction) befindet, spielen kleinere Änderungen exogener Größen, auch durch politische Maßnahmen, kaum eine Rolle und beeinflussen das langfristige Systemverhalten nicht wesentlich, auch wenn starke kurzfristige Effekte möglich sind. Allerdings kann es auch sein, dass politische Maßnahmen auch langfristig sehr große Auswirkungen haben, entweder, wenn sie das System aus dem Einzugsbereich eines Attraktors in denjenigen eines anderen bringen oder, wenn das System sich zunächst außerhalb von Attraktoreinzugsbereichen befindet. Komplexe adaptive Systeme können also sehr robust gegenüber exogenen Einflüssen sein oder auch sehr sensibel auf diese reagieren.

\section{Bedeutung für die Makroökonomik}

Die bisher abstrakten Ausführungen sollen nun konkret auf die Makroökonomik angewandt werden. Dazu zeige ich, worin sich die Aussagen der Mainstream-Makroökonomik grundlegend von denen einer Komplexitätsmakroökonomik unterscheiden.

Ein zentraler und grundlegender Unterschied besteht darin, wo die Ursachen für makroökonomische Dynamiken verortet werden. DSGE-Modelle erklären ökonomische Fluktuationen durch unvorhersehbare exogene Ereignisse. Im Grunde findet dadurch aber keine Erklärung statt, weil diese Ereignisse als Zufallsgrößen modelliert werden, de- 
ren Ursprung unbekannt ist (vgl. Blanchard 2009). In komplexen adaptiven Systemen kann es auch Zufallseinflüsse geben. Jedoch haben diese Systeme auch ohne regelmäßig auftretende Schocks eine komplizierte endogene Dynamik, die durch die Interaktion der Agenten und ihr Lernund Anpassungsverhalten ausgelöst wird. Dadurch können prinzipiell Mechanismen beschrieben werden, durch die Fluktuationen nicht nur ausgelöst, sondern auch vorangetrieben werden. Jedoch ist es dann nicht mehr so einfach, von Ursache und Wirkung zu sprechen, weil in einem nicht-linearen komplexen adaptiven System jede Variablenänderung vielfältige Anpassungen anstößt, die ihrerseits wiederum neue Auslöser für weitere Verhaltensanpassungen sind. Solche Feedbackprozesse heben auch die Proportionalität zwischen der Stärke von exogenen Änderungen und endogenen Reaktionen auf, die für DSGE-Modelle typisch ist.

In der Mainstream-Makroökonomik ist die Struktur einer Ökonomie in der Regel unveränderlich, d.h. das Verhalten von Haushalten, Unternehmen und staatlichen Akteuren, technologische und institutionelle Zusammenhänge und Gleichgewichtsbedingungen können durch feste funktionale Formen beschrieben werden. Dieses Weltbild ist mechanistisch und legt eine große Plan- und Steuerbarkeit der Ökonomie nahe. Prinzipiell können solche Ökonomien durch Gleichgewichte und deren Änderungen analysiert werden. In der Komplexitätssicht ist dies anders. Eine komplexe Ökonomie muss nach einer exogenen Änderung nicht zum Ausgangsgleichgewicht zurückkehren, sondern kann ihre Struktur und damit auch ihr Verhalten qualitativ verändern. Solche Systemänderungen können unter Umständen sehr plötzlich und überraschend auftreten. Auch ist es wenig sinnvoll, die Wirtschaftspolitik oder Institutionen ${ }^{13}$ als exogen von der Ökonomie im engeren Sinne zu betrachten. Durch das adaptive Verhalten der verschiedenen Agenten werden Institutionen verändert und endogene Politikwechsel eingeleitet. In Kombination mit der geschilderten Pfadabhängigkeit und Irreversibilität hat dies tiefgreifende wirtschaftspolitische Implikationen. Wirtschaftspolitische Maßnahmen müssen nicht zu allen Zeiten und unter allen Zuständen des Systems immer gleich wirken. Außerdem mag es sein, dass das System nicht durch die bloße Rücknahme politischer Maßnahmen in seinen Ausgangszustand zurückversetzt werden kann. Eine Änderung des Steuersystems oder der

13 Nach North (1991) werden Institutionen hier als von Menschen gestaltete Beschränkungen verstanden, die politische, ökonomische und soziale Interaktionen strukturieren. Sie können sowohl aus informellen Beschränkungen bestehen (z. B. Tabus, Sitten, Traditionen) als auch als formalen Regeln (z. B. Gesetze, Eigentumsrechte). geldpolitischen Ausrichtung wird dann nicht nur durch eine Parameter- oder Variablenänderung erfasst, sondern hat Auswirkungen auf strukturelle Zusammenhänge. Beispielsweise ist es denkbar, dass durch bestimmte Steuern ganze Sektoren oder Arten von Unternehmen neu entstehen oder auch verschwinden, was durch eine Rücknahme der Steuern nicht umgekehrt wird.

Aus dem Gesagten folgt auch ein grundlegender Unterschied im Umgang mit Unsicherheit. Das mechanistische Weltbild der neoklassischen Ökonomik legt nahe, dass es eine prinzipiell feste Struktur einer Volkswirtschaft gibt, die erkennbar ist und daher auch für Prognosen verwendet werden kann. Zwar enthalten alle Beobachtungen über die Ökonomie immer auch Zufallseinflüsse, die nicht vorhergesehen werden können, aber letztlich gibt es eine Tendenz zu einem bestimmbaren Gleichgewicht. Daher ist auch die Theorie rationaler Erwartungen innerhalb dieses Gedankengebäudes vollkommen folgerichtig. Die Komplexitätsökonomik hingegen betont die fundamentale Unsicherheit, der sowohl die einzelnen Akteure in einer Ökonomie als auch die Wirtschaftsforscher ausgesetzt sind. Ökonomische Prognosen sind dann nur in Wahrscheinlichkeiten und auch nur in kurzen Fristen möglich. Dies würden zwar auch die meisten Mainstream-Ökonomen so sehen, jedoch ergibt sich aus dem Gesagten, dass bei fundamentaler Unsicherheit und der Möglichkeit endogener Strukturveränderungen die Fehlerwahrscheinlichkeit jeder Prognose größer sein muss als in Modellen, in denen die Unsicherheit lediglich durch additive stochastische Schocks abgebildet wird.

Wenn man von Selbstorganisation und Emergenz ausgeht, ist klar, dass Konzepte wie repräsentative Agenten und rationale Erwartungen zentrale Systemeigenschaften nicht erfassen können. Das Konzept des repräsentativen Agenten löst das Aggregationsproblem mit dem Trick, dass per Annahme die Eigenschaften des Aggregats identisch mit denen der bzw. des Agenten sind. Dies steht geradezu im Widerspruch zum Phänomen der Emergenz, bei der das Aggregat andere Eigenschaften hat als jeder einzelne Agent. Ebenso widerspricht die Theorie der rationalen Erwartungen der Idee der spontanen Herausbildung einer Systemstruktur, die nicht einfach aus der Kenntnis der Eigenschaften der Systemelemente allein prognostiziert werden kann.

\section{Evidenz}

In diesem Abschnitt möchte ich verschiedene Arten empirischer Evidenz vorstellen, die mit der Mainstream-Makroökonomik nur schwer zu vereinbaren sind, aber völlig im 
Einklang mit der Komplexitätsökonomik stehen. ${ }^{14} \mathrm{Zu}$ nächst soll dazu auf mikroökonomische Evidenz zum Verhalten von Individuen eingegangen werden, anschließend auf makroökonomische Phänomene.

Makroökonomische Mainstream-Modelle beruhen sehr stark auf der Annahme repräsentativer Agenten mit rationalen Erwartungen. Dazu im klaren Widerspruch steht der empirische Befund, dass es zu jeder Zeit erhebliche Heterogenität in den Erwartungen von Wirtschaftssubjekten gibt (siehe z. B. Carroll 2003, Mankiw et al. 2003, Souleles 2004, Capistrán und Timmermann 2009). Die experimentelle Learning-to-Forecast-Literatur (siehe Hommes 2011) zeigt, dass Wirtschaftssubjekte im Labor meist einfache Heuristiken für die Erwartungsbildung verwenden und häufig zwischen verschiedenen Heuristiken wechseln. Dies ist ein Beispiel nicht nur für begrenzt rationales, sondern auch für adaptives Verhalten, weil Agenten ihr Verhalten an das der anderen bzw. an das Feedback aus dem Aggregat anpassen.

Ein Grundprinzip der neoklassischen (Makro-)Ökonomik ist, dass Wirtschaftssubjekte konstante, exogen gegebene Präferenzen haben und Verhalten durch Änderungen von Preisen und Einkommen, aber nicht durch Präferenzänderungen erklärt wird. Auch dies steht im Widerspruch zur empirischen Evidenz. ${ }^{15}$ In der Finance-Literatur ist seit längerem bekannt, dass es schwierig ist, Volatilitäten und Risikoprämien von sicheren und riskanten Wertpapieren mit einer gegebenen Risikoaversion zu erklären (siehe Mehra und Prescott 1985). Stattdessen passen Modelle mit zeitvariabler Risikoneigung wesentlich besser zu den Daten (siehe Campbell und Cochrane 1999, 2000, Maio 2009). Guiso et al. (2013) zeigen sowohl ökonometrisch anhand von Umfragedaten als auch experimentell, dass private Investoren ihre Risikoneigung ändern, wenn sie mit beängstigenden Ereignissen wie der Finanzkrise 2008 konfrontiert werden. Ähnliche Ergebnisse sind aus dem Versicherungsverhalten von Haushalten nach Katastrophen wie Überschwemmungen oder Erdbeben bekannt (siehe van den Berg et al. 2009, Cameron und Shah

14 Diese Aussage ist natürlich pauschal. Es lassen sich vermutlich immer Beispiele für neoklassische Modelle finden, die zu den genannten empirischen Fakten passen. Jedoch müssen solche Modelle Annahmen machen, die von denen des Mainstreams abweichen und nur bedingt zum neoklassischen Theoriegebäude passen. Außerdem ist es im Rahmen der neoklassischen Methode in der Regel nur möglich, einzelne Annahmen aufzuweichen, um bestimmte Phänomene abzubilden.

15 Und, wie einer der Gutachter anmerkte, auch im Widerspruch zur Lebenserfahrung. Es ist daher zumindest erstaunlich, dass der neoklassisch geprägte Mainstream die Beweislast für veränderliche oder kontextabhängige Präferenzen der Verhaltensökonomik aufbürdet.
2013). Malmendier und Nagel (2011) weisen nach, dass die Risikoneigung und die Bereitschaft zur Übernahme von Risiken beeinflusst werden, wenn Menschen in jungen Jahren geringe Erträge auf Aktienmärkten erfahren haben. Guiliano und Spilimbergo (im Erscheinen) zeigen, dass Präferenzen für Umverteilung und Selbstwirksamkeitsüberzeugungen durch die Erfahrung einer Rezession im frühen Erwachsenenalter beeinflusst werden. Über diesen Kanal haben Rezessionen auch Auswirkungen auf späteres Wahlverhalten. Die ökonomischen Bedingungen beeinflussen aber nicht nur Präferenzen, Einstellungen und Verhaltensweisen von privaten Haushalten, sondern auch von Unternehmensmanagern, wie Malmendier et al. (2011) und Schoar und Zuo (2014) zeigen. Neben der Endogenität von Präferenzen zeigen diese Beispiele auch die Pfadabhängigkeit makroökonomischer Entwicklungen.

Ebenso grundlegend in der Neoklassik ist die Annahme, dass Individuen Entscheidungen unabhängig von anderen auf der Basis eigener Kosten-/Nutzenerwägungen treffen. Brock und Durlauf (2006) zitieren eine Reihe von empirischen Studien, die zeigen, dass individuelles Verhalten durch soziale Interaktion mit anderen entscheidend mitbestimmt wird. Dafür gibt es z. B. Evidenz im Zusammenhang mit Verbrechen, Inanspruchnahme von Sozialleistungen, Beschäftigungsentscheidungen oder Rauchen. Soziale Interaktion kann individuelles Verhalten z.B. durch soziale Normen beeinflussen, die regeln, welches Verhalten von einer Gruppe akzeptiert oder abgelehnt wird. In Situationen mit multiplen Gleichgewichten können solche sozialen Normen die Koordination auf ein gemeinsames Verhalten erreichen. Da sich Normen auch endogen ändern können, entstehen positive Feedbackeffekte, durch die kleine exogene Schocks durch Interaktion von Individuen verstärkt werden. Ein Beispiel dafür ist, dass durch einen negativen Schock dauerhaft die Arbeitslosenquote in einer Region ansteigen kann. Wenn viele Personen zur gleichen Zeit ihre Beschäftigung verlieren, können dadurch soziale Normen über die Erwerbstätigkeit geändert werden. Somit kann es für Arbeitslose sozial akzeptabel werden, dauerhaft Schwarzarbeit anzubieten, so dass es zu einem permanenten negativen Effekt auf das Arbeitsangebot im offiziellen Sektor kommt. Sowohl Brock und Durlauf (2006) als auch Akerlof (2007) argumentieren, dass die Berücksichtigung sozialer Normen wichtig für das Verständnis makroökonomischer Phänomene ist. Die Berücksichtigung direkter und indirekter sozialer Interaktion ist in herkömmlichen ökonomischen Modellen schwierig, nicht aber in agentenbasierten Modellen, für die sie charakteristisch ist.

Die makroökonomische Geschichte der letzten Jahrzehnte liefert zahlreiche Beispiele für Phänomene, die aus 
der Sicht der Komplexitätsökonomik völlig natürlich erscheinen, in der Mainstreamökonomik aber Anomalien sind.

Der eindrucksvollste und aktuellste Fall ist natürlich die globale Finanzkrise von 2007-2009, die für die meisten Markteilnehmer und Ökonomen völlig überraschend ausbrach. Die Standard-Makroökonomik arbeitet noch immer daran $\mathrm{zu}$ verstehen, wieso diese Krise so plötzlich ausbrach, wieso sie so gravierend war und weshalb ihre Folgen so lange andauern. Nichts davon ist aus Sicht der Komplexitätsökonomik ungewöhnlich. Wie bereits dargestellt, können nicht-lineare dynamische Systeme Bifurkationen haben, an denen sich bei einer kleinen Variablenänderung der Zustand des Systems plötzlich und qualitativ sehr stark ändert. Auch in der Mainstream-Literatur wird mittlerweile häufig auf Selbstverstärkungsprozesse verwiesen, um zu erklären, wie aus verhältnismäßig kleinen Problemen im US-Subprime-Markt ein BeinaheKollaps des globalen Finanzsystems und eine weltweite Rezession entstehen konnten (siehe Brunnermeier 2009). Wenn es Pfadabhängigkeit und Irreversibilität gibt, ist es auch nicht überraschend, dass die globale Wirtschaft auch fünf Jahre nach der Krise deren Folgen nicht bewältigt und zurück zum ursprünglichen Gleichgewicht gefunden hat.

Ähnliche Argumente können auch für die noch immer andauernde Euro-Krise angeführt werden. So ist es schwierig zu sehen, worin der gravierende exogene Schock bestanden hat, der im Frühjahr 2010 dazu führte, dass die Risikoprämien griechischer Staatsanleihen exorbitant anstiegen und Griechenland beinahe in den Staatsbankrott trieben. Fundamental dürfte sich in Griechenland in dieser Zeit wenig geändert haben. Es erscheint plausibler, dass es zu einem Stimmungseffekt kam, der durch Feedbackeffekte enorm verstärkt wurde.

Sowohl für die globale Finanzkrise als auch für die Krise im Euroraum werden häufig geänderte Risikoeinstellungen und search for yield von Finanzinvestoren als Ursachen genannt (Buch et al. 2014). Diese können durch geldpolitische Entscheidungen, Innovationen im Risikomanagement, durch institutionelle und regulatorische Änderungen und Interaktionseffekte zwischen Investoren erklärt werden. Solche Erklärungen findet man in der angewandten Literatur (Rajan 2005, Nirei et al. 2012, Altunbas et al. 2014), jedoch fällt es der makroökomischen Standardtheorie schwer, sie zu formalisieren. Postkeynesianer wie Hyman Minsky jedoch vertreten schon lange Krisentheorien, nach denen Phasen der makroökonomischen und finanziellen Stabilität - wie die große Moderation vor der Finanzkrise - u. a. durch geändertes Risikoverhalten finanzielle Instabilität bedingen.
Auch weitere Phänomene passen gut zur Interpretation von Ökonomien als komplexe adaptive Systeme. So gibt es Evidenz für regulatorische Zyklen (vgl. Clarke 2004, McDonnell 2013), wonach nach Finanzkrisen die Finanzmarktregulierung deutlich verschärft wird. Wenn es längere Zeit nicht zu Krisen kam, gibt es politischen Druck die Finanzmärkte wieder zu deregulieren, wodurch in vielen Fällen wieder Krisen ausgelöst werden (vgl. Allen und Gale 2009). Das Verhalten des Regulators scheint in diesem Zusammenhang eher begrenzt rational und adaptiv als vollkommen rational zu sein, und es ist ohne Zweifel endogen.

Im Zusammenhang mit der Asienkrise von 1997/98 wird zuweilen das Argument vorgebracht, dass die Ansteckung der verschiedenen Länder mit der Krise nur schwer durch Handels- oder Finanzverflechtungen zu erklären ist. Vielmehr scheint es einen psychologischen Effekt unter den Finanzinvestoren gegeben $\mathrm{zu}$ haben, die, nachdem die Krise in Thailand ausgebrochen war, von einer Panik und einem Herdentrieb erfasst wurden und ohne $\mathrm{zu}$ differenzieren Kapital aus allen Ländern dieser Region abzogen (Kaminsky et al. 2004).

Das Phänomen der Jobless recovery nach Krisen ist in Standard-Modellen ebenfalls nicht leicht zu erklären (vgl. Schmitt-Grohé und Uribe 2012). Für komplexitätsökonomische Modelle mit endogenen Arbeitsangebotsreaktionen oder sektoralem Strukturwandel könnte es hingegen leichter abzubilden sein.

Ein anderes Beispiel für eine Irreversibilität im makroökonomischen Kontext ist das Phänomen der Dollarisierung. Wenn ein Land durch schlechte Geldpolitik hohe Inflation erzeugt und damit das Vertrauen in seine Währung erschüttert, kommt es häufig dazu, dass die Wirtschaftssubjekte die nationale Währung gegen eine stabilere Auslandswährung, häufig den Dollar, substituieren. Dies geschah z. B. von 1998 bis 2000 in Ecuador. Selbst wenn die Geldpolitik durch einen radikalen Strategiewechsel die Inflationsprobleme in den Griff bekommt, ist es für ein Land häufig sehr schwierig diese Währungssubstitution wieder umzukehren (vgl. Reinhart et al. 2014). Ähnlich könnte es sein, wenn ein Land in einer Schuldenkrise exzessive Sparpolitik betreibt und damit eine längerfristige Depression auslöst. Selbst wenn die Fiskalpolitik irgendwann wieder expansiver wird, kann die zwischenzeitliche Depression die Ökonomie fundamental verändert haben, so dass es keine Rückkehr zu einem ursprünglichen Zustand gibt.

All diese Beispiele beweisen natürlich nicht, dass eine Volkswirtschaft ein komplexes adaptives System ist. Eine Auflistung von Einzelbeispielen ist ein induktives Vorgehen, durch das prinzipiell kein Wahrheitsbeweis 
möglich ist. Allerdings kann bereits durch einzelne Gegenbeispiele eine Aussage falsifiziert werden. In diesem Sinne sei noch einmal betont, dass die genannte Evidenz gegen die üblichen Annahmen der makroökonomischen Mainstreammodelle spricht. Die ökonomische Profession hat eine Tendenz, lange an den Standardannahmen perfekter Rationalität und unabhängiger Entscheidungen bei gegebenen Präferenzen festzuhalten und beobachtete Anomalien durch Zusatzannahmen zu erklären. Dabei wären Erklärungen, die z.B. begrenzte Rationalität oder normengeleitetes Verhalten zulassen, häufig einfacher (vgl. Akerlof 2007). Im Sinne von Ockhams Rasiermesser wäre es gute wissenschaftliche Praxis, in solchen Fällen komplexitätsökonomische Ansätze gegenüber denen der neoklassischen Ökonomik zu bevorzugen (vgl. auch Simon 1976).

\section{Implikationen für die Wirtschaftspolitik}

Es sollte deutlich geworden sein, dass die Komplexitätsökonomik ein fundamental anderes Verständnis makroökonomischer Mechanismen hat als die Mainstream-Makroökonomik. Folglich stellt sich die Frage, ob und inwiefern sie andere wirtschaftspolitische Empfehlungen gibt. Diese Frage muss letztlich anhand von konkreten Beispielen diskutiert werden, was im Rahmen dieses Essays nicht möglich ist. Allerdings können auf der Grundlage der vorherigen Ausführungen einige grundsätzliche Überlegungen angestellt werden.

Eine wichtige Botschaft ist, dass Idiosynkrasien wichtig sind und ein politisches Rezept nicht für alle Zeiten, alle Länder und alle Gesellschaften funktionieren muss. Die neoklassische Ökonomik reduziert das Wirtschaftssystem auf Präferenzen, Technologien, Marktstrukturen und gesamtwirtschaftliche Identitäten und verbindet dies mit der Annahme universaler Rationalität und Nutzenund Gewinnmaximierung. Dieser Denkansatz wird auf alle Ökonomien angewendet, Unterschiede gibt es bestenfalls in Modellparametern. In der Komplexitätsökonomik ist das ökonomische System in ein breiteres Gesellschaftssystem eingebettet. In verschiedenen Gesellschaften kann es sehr unterschiedliche Präferenzen, Institutionen und soziale Normen geben, die das Verhalten der Agenten maßgeblich beeinflussen und sich im Zeitablauf endogen ändern können. Daraus folgt, dass eine wirtschaftspolitische Maßnahme, die zu einem Zeitpunkt eine bestimmte Wirkung hatte, diese Wirkung zu einem anderen Zeitpunkt nicht mehr haben muss. Dasselbe gilt für die Übertragbarkeit zwischen Ländern. Wenn es keine einfachen Patentrezepte für die Wirtschaftspolitik gibt, die immer und überall richtig sind, folgt daraus, dass wirtschaftspolitische Empfehlungen im komplexitätsökonomischen Ansatz weniger eindeutig, gewiss und apodiktisch sein können als im Standardansatz (vgl. Elsner et al. 2014, S. 504).

Mit diesem Punkt eng verbunden ist das Problem, dass empirische Evidenz aus der Vergangenheit oder Länderquerschnittsstudien nur begrenzte Aussagekraft für zu erwartende zukünftige Effekte wirtschaftspolitischer Maßnahmen hat. Empirische Forschung kann naturgemäß nur vergangene Zusammenhänge aufdecken, die aber nicht stabil sein müssen. Das bedeutet nicht, dass eine Analyse solcher Zusammenhänge nicht informativ ist. Allerdings muss man sich klarmachen, wie begrenzt der Nutzen solcher Studien für die Abschätzung von zukünftigen Politikfolgen ist. Selbst wenn man feststellen könnte, wie groß z. B. der Staatsausgabenmultiplikator für das BIP in einem Land in der Vergangenheit war (was notorisch schwierig ist, vgl. Roos 2007), sollte man aus Sicht der Komplexitätsökonomik nicht darauf vertrauen, dass er konstant ist. Die Komplexitätsökonomik würde empfehlen, z.B. auf der Grundlage von agentenbasierten Modellen verschiedene Szenarien zu simulieren, die sich bei alternativer Kalibrierung des Modells auf einen gegenwärtigen Zustand ergeben könnten. Damit wäre es möglich abzuschätzen, innerhalb welcher Grenzen sich bestimmte Effekte möglicherweise bewegen und mit welchen Wahrscheinlichkeiten sie auftreten. Dies ist anders als in der Standardmakroökonomik, in der natürlich auch Simulationen mit kalibrierten Modellen durchgeführt werden. Der Unterschied besteht darin, dass Gleichgewichtsmodelle bei identischer Kalibrierung immer dieselben Anpassungspfade hin zu denselben Gleichgewichten haben, während dies in komplexen adaptiven Modellen nicht der Fall sein muss.

Die Eigenschaften komplexer adaptiver Systeme stellen die Möglichkeit von sinnvollen theoriegestützten Prognosen über zukünftige Ereignisse grundsätzlich in Frage. Zwar betonen auch neoklassische Ökonomen immer die Beschränkungen von Prognosen angesichts unvorhersagbarer Schocks. Dennoch wird im Allgemeinen nicht bezweifelt, dass Prognosen auf der Grundlage linearer oder linearisierter Gleichgewichtsmodelle sinnvoll sind. Die Komplexitätsökonomik fordert die Ökonomen zu mehr Bescheidenheit hinsichtlich ihrer Prognosefähigkeiten auf und wehrt auch wirtschaftspolitische Ansprüche auf zuverlässige Mittelfristprognosen ab. Die Komplexitätsökonomik legt nahe, dass Ökonomen und auch die Gesellschaft akzeptieren müssen, dass konkrete Krisenprog- 
nosen ähnlich wie Erdbebenprognosen ${ }^{16}$ prinzipiell kaum möglich sind und es bestenfalls nur kurzfristige Frühwarnungen geben kann.

Die komplexitätsökonomische Sicht betont, dass das Wirtschaftssystem gesellschaftlich eingebettet ist. Selbst wenn in Modellen immer nur ein Ausschnitt der Realität abgebildet werden kann, muss für eine verlässliche Analyse berücksichtigt werden, dass es zwischen ökonomischen und gesellschaftlichen Prozessen eine Wechselwirkung gibt. Demnach sollten wirtschaftspolitische Fragen nicht aus einer isolierten rein ökonomischen Perspektive betrachtet werden, sondern erfordern einen breiteren Blick auf soziale Prozesse, die durch wirtschaftspolitische Maßnahmen möglicherweise ausgelöst werden. Bei einer $\mathrm{zu}$ engen ökonomischen Betrachtungsweise kann es dazu kommen, dass Wirtschaftssubjekte nicht in der erwarteten Weise auf Politikmaßnahmen reagieren. Damit soll nicht gesagt sein, dass ökonomische Anreize unwichtig für die Reaktion der Wirtschaftssubjekte auf politische Maßnahmen sind. Jedoch werden ökonomische Anreize unter Umständen von anderen Faktoren überlagert, die in der neoklassischen Analyse keine Rolle spielen. Ein Beispiel dafür ist die Einführung des Kraftstoffs E10 im Jahr 2011 in Deutschland, die politisch gewollt war. Die Mineralölindustrie erwartete bei der Einführung von E10 mittelfristig einen Marktanteil von 80 Prozent für diese Benzinsorte, aber seit 2013 stagniert der Marktanteil bei etwa 15 Prozent, obwohl E10 billiger ist und es keine Evidenz für die befürchteten Risiken für Motoren gibt. ${ }^{17}$ Aus einer rein neoklassischen Perspektive, nach der Autofahrer die Kosten, den Nutzen und die möglichen Risiken dieses Kraftstoffs gegen diejenigen von Substituten wie dem Kraftstoff E5 abwägen sollten, ist die relativ geringe Nachfrage schwer zu verstehen. Ein wichtiger Faktor ist sicher die Hysterie über mögliche Motorschäden, die bei der Einführung dieses Kraftstoffs auch durch die Medien verbreitet wurde.

Aus wirtschaftspolitischer Sicht ist auch wichtig, dass komplexe adaptive Systeme in der Regel endogen Ungleichheit erzeugen, selbst bei zunächst identischen Agenten. Politische Diskurse werden durch Ungleichheitsfragen dominiert, und häufig geht es bei politischen Maßnahmen weniger um die Beseitigung von Ineffizienzen als um die

16 Vgl. die Debatte über die Vorhersagbarkeit von Erdbeben in Nature (1999) unter: http://www.nature.com/nature/debates/earth quake/.

17 http://www.spiegel.de/auto/aktuell/biosprit-e10-keine-pro bleme-aber-mauer-absatz-a-995651.html, http://www.welt.de/wirtschaft/energie/article132625779/Von-Motors chaeden-durch-E10-Betankung-keine-Spur.html.
Lösung von Verteilungskonflikten. Die neoklassische Ökonomik hat auf Grund der Verwendung von repräsentativen Agenten wenig zu Verteilungsfragen zu sagen. Wenn in solchen Modellen Ungleichheit thematisiert wird, wird sie häufig als exogen angenommen oder folgt direkt aus individuellen Unterschieden, z.B. in der Begabung oder dem Bildungsniveau. Modelle, in denen Ungleichheit endogen auch bei identischen Agenten entstehen kann, können zu anderen Einsichten und politischen Schlussfolgerungen führen.

Während die neoklassische Gleichgewichtsökonomik von der klassischen Mechanik inspiriert ist, orientiert sich die Komplexitätsökonomik eher an der Evolutionstheorie. Die Mechanik sucht nach festen Ursache-Wirkungsbeziehungen auf der Grundlage unveränderlicher Naturgesetze. Die Evolution ist grundsätzlich prozessorientiert, offen und nicht zielgerichtet, so dass fundamentale Unsicherheit herrscht und die zukünftige Entwicklung nicht vorhergesagt werden kann. Überraschungen, Wandel und Krisen sind daher der Normalfall. Der Wirtschaftspolitiker ist dann weniger ein Ingenieur, der eine Maschine durch das Drücken der richtige Knöpfe steuern kann, als ein Biologe, der durch begründetes Experimentieren versucht, einen Prozess in einer gewünschten Richtung zu beeinflussen. Das bedeutet, dass in der Wirtschaftspolitik nicht nach Patentrezepten und einfachen, immer gültigen Lösungen gesucht, sondern vielmehr mit verschiedenen Ansätzen experimentiert werden sollte. Die Vorstellung, dass die Geldpolitik mindestens ebenso sehr eine Kunst wie eine Wissenschaft ist, ${ }^{18}$ kommt dieser Auffassung bereits recht nahe. Allgemein kann gesagt werden, dass Wirtschaftspolitik stärker induktiv vorgehen sollte und bereit sein muss, Maßnahmen, die temporär wirksam sind und ihren Zweck erfüllen, auch wieder zurückzunehmen, wenn sich neue Erkenntnisse ergeben oder sich die Umstände geändert haben (vgl. Elsner et al. 2014, S. 504).

Aus dem Gesagten folgt, dass die Komplexitätsökonomik eine große Skepsis gegenüber zentralplanerischen Vorstellungen von Wirtschaftspolitik nahelegt. Es dürfte unmöglich sein, in komplexen adaptiven Systemen Aussagen über optimale Politiken jeglicher Art - sei es optimale Steuerpolitik, optimale Fiskalpolitik oder optimale Geldpolitik - zu machen. Sobald man sich außerhalb des wohldefinierten neoklassischen Modellrahmens bewegt, ist weder klar, was eine First-Best-Policy ist, noch dass man sich ihr über einen Second-Best-Ansatz annähern

18 vgl. Dell'Arricia und Habermeier: http://blog-imfdirect.imf.org/2 014/04/07/the-evolution-of-monetary-policy-more-art-and-less-sci ence/. 
kann (vgl. Elsner et al. 2014, S. $501 \mathrm{ff}$.). Daher besteht bei zentralistischer Wirtschaftspolitik immer auch ein großes Risiko von Fehlentscheidungen mit schwerwiegenden Folgen auf Grund der großen Reichweite zentraler Politikmaßnahmen. In dieser Hinsicht wird das Erbe von Hayek und der österreichischen Schule in der Komplexitätsökonomik deutlich. Man muss nicht so weit gehen, staatlichen Akteuren grundsätzlich zu misstrauen und grundsätzlich Märkten den Vorzug gegenüber staatlichen Regelungen zu geben. Jedoch kann man aus dieser Denkweise ein Plädoyer für eine weitreichende Dezentralität wirtschaftspolitischer Entscheidungen ableiten. Dezentralität kann dabei bedeuten, dass Entscheidungen von lokalen Gebietskörperschaften getroffen werden, aber auch, dass andere gesellschaftliche Gruppen wie Nichtregierungsorganisationen aktiv an der Lösung gesellschaftlicher Probleme beteiligt sind. Eine dezentrale Wirtschaftspolitik, bei der viele Akteure parallel nach der Lösung ähnlicher Probleme suchen, ist dann auch ein Entdeckungsverfahren in einer von fundamentaler Unsicherheit und ständigem Wandel geprägten Welt. Dezentrale Akteure können voneinander lernen, lokale Informationen möglicherweise besser verarbeiten und verursachen bei falschen Entscheidungen vermutlich weniger Schaden als ein zentraler Entscheider.

Aus dem Gesagten folgt aber keineswegs, dass die Wirtschaftspolitik keine wichtigen Funktionen hätte und staatliche Akteure sich aus dem Wirtschaftsgeschehen heraushalten sollten. Allerdings spricht die Komplexitätsökonomik eher für eine schlankere Politik mit weniger direkten Interventionen, z. B. in Form von staatlicher Bereitstellung von Gütern und Dienstleistungen oder Anreizsetzung durch Steuern oder Subventionen. Vielmehr besteht eine zentrale Aufgabe der Wirtschaftspolitik darin, einen geeigneten institutionellen Rahmen zu finden, der die Koordination und Kooperation privater Akteure erleichtert. Mit nichtpekuniären Instrumenten können Kooperationsblockaden aufgelöst werden (vgl. Elsner et al. 2014). Colander und Kupers (2014) sprechen davon, dass der Staat die „Ökostruktur“ der Wirtschaftssubjekte beeinflussen muss. Durch geeignete regulatorische Rahmenbedingungen kann der Staat indirekt Präferenzen und soziale Normen beeinflussen und damit gesellschaftlich wünschenswerte Ergebnisse zwar nicht selbst herbeiführen, aber ihre Entstehung begünstigen. Da die Kooperation privater Akteure am besten in überschaubaren Gruppen funktioniert, sollte der Staat die Entstehung entsprechender Institutionen auf lokaler, regionaler oder industrieller Ebene fördern. Eine wichtige Funktion solcher Netzwerke ist z. B. der Austausch von Informationen. Elsner et al. (2014) bezeichnen eine solche Politik als meso-ökonomische
Wirtschaftspolitik, die die tiefe Struktur einer Volkswirtschaft beeinflusst.

Wie oben diskutiert wurde, können komplexe adaptive Systeme strukturelle Instabilitäten aufweisen, die zu endogenen Krisen und Zustandsänderungen führen können. Eine wichtige wirtschaftspolitische Aufgabe ist dann, solche strukturellen Instabilitäten nach Möglichkeit durch entsprechende Regulierung zu beseitigen. Viele komplexitätsökonomische Arbeiten zeigen, dass regulatorische Maßnahmen in Netzwerken auf komplizierte Weise zusammenwirken können. So ist es das Ziel des CRISIS-Projekts ${ }^{19}$, innerhalb eines komplexitätsökonomischen Rahmens die inhärente Instabilität des Finanzsystems zu erforschen und aus den gewonnenen Erkenntnissen Empfehlungen für eine Finanzarchitektur und Finanzmarktregulierung herzuleiten, die das Finanzsystem wirkungsvoll stabilisieren.

Neben dieser indirekten Form der Wirtschaftspolitik kann in komplexen Systemen auch eine direkte staatliche Intervention begründet werden, wenn sich ein Wirtschaftssystem in einer evolutorischen Falle befindet. Damit ist ein ineffizientes Gleichgewicht gemeint, aus dem sich die privaten Agenten nicht von allein befreien können. Durch geeignete staatliche Maßnahmen könnte das System dann in den Einzugsbereich eines besseren Attraktors gebracht werden. Beispiele für solche evolutorische Fallen sind sogenannte Armutsfallen (vgl. Kraay und McKenzie 2014), technologische Lock-ins (vgl. Arthur 1989) oder auch gesellschaftliche Ungleichheit und Segregation (vgl. Durlauf 1998).

\section{Fazit}

In diesem Aufsatz habe ich skizziert, welche Sicht die Komplexitätsökonomik in Bezug auf Eigenschaften und Funktionsweise von Ökonomien hat und wie sich diese Sicht von der den ökonomischen Mainstream dominierenden Neoklassik unterscheidet. Insbesondere wurde gezeigt, dass die Komplexitätsökonomik das Potential hat, einige gravierende Probleme der Mainstream-Makroökonomik zu lösen.

Die Komplexitätsökonomik beginnt gerade erst sich zu entwickeln. Mit Hilfe von agentenbasierten Modellen werden einige ihrer Prinzipien bereits in der Makroökonomik und anderen Bereichen angewandt. Dieser Aufsatz soll daher auch ein Anstoß sein, dieses neue Forschungsfeld ernst zu nehmen und voranzutreiben. Da die Komple-

19 http://www.crisis-economics.eu/. 
xitätsökonomik nicht davon ausgeht, dass man ökonomische Systeme von ihrem gesellschaftlichen Umfeld trennen kann, wird solche Forschung eine Zusammenarbeit mit anderen Disziplinen wie der Soziologie oder der Politikwissenschaft erfordern. Dazu ist sicher ein Mentalitätswandel in der Volkswirtschaftslehre erforderlich. Ein solcher Mentalitätswandel muss nicht unbedingt in einen Paradigmenwechsel münden, jedoch sollte er $\mathrm{zu}$ mehr Offenheit für von der neoklassischen Denkweise verschiedene Forschungsrichtungen und interdisziplinärer Arbeit führen.

Die Komplexitätsökonomik impliziert aber auch einen Abschied von tradierten Denkweisen der Wirtschaftspolitik und der wirtschaftspolitischen Beratung. Wenn man die Ökonomie als komplexes adaptives System begreift, ist es sehr fraglich, ob es eine optimale und vielleicht sogar auch noch einfache Wirtschaftspolitik geben kann. Dies könnte z.B. für den Glauben an die Steuerbarkeit einer Volkswirtschaft durch geldpolitische Rezepte wie Inflation targeting oder einfache Regeln wie die TaylorRegel gelten oder auch ideologisch geprägte Forderungen nach „mehr Markt“ oder „mehr Staat“. Wie eine gute Wirtschaftspolitik - was auch immer darunter zu verstehen ist - aus einer komplexitätsökonomischen Perspektive aussieht, kann an dieser Stelle nicht beantwortet werden. Dazu ist noch viel mehr Forschung erforderlich. Diese Forschung wird möglicherweise noch weniger klare Antworten auf wirtschaftspolitische Fragen geben als es die bisherige Mainstream-Forschung tut. Nicht-lineare dynamische Systeme sind wesentlich schwieriger $\mathrm{zu}$ analysieren als lineare Modelle, haben schwer durchschaubare Ursache-Wirkungsbeziehungen und erlauben oft keine eindeutigen Prognosen. Dies kann aber kein Vorwurf an solche Modelle sein. Wenn die Volkswirtschaft ein komplexes adaptives System ist, sollte sie auch durch angemessene Modelle beschrieben werden, selbst wenn diese im Vergleich $\mathrm{zu}$ den eleganten und relativ einfach anzuwendenden Modellen der Neoklassik schwerer zu durchschauen sind und weniger klare Aussagen machen. Auch wenn diese scheinbaren Beschränkungen komplexitätsökonomischer Modelle für den Anwender zunächst enttäuschend klingen, kann man sie auch positiv sehen. Alle Modelle sind nur Vereinfachungen der Realität und können immer nur Anhaltspunkte über mögliche Kausalzusammenhänge, zukünftige Entwicklungen und Wirkungen von politischen Maßnahmen geben. Die Komplexitätsökonomik kann dazu beitragen, diesen Sachverhalt nicht $\mathrm{zu}$ vergessen und deutlich machen, dass die ökonomischen Modelle und Daten interpretiert werden müssen und ihre Anwendung subjektive Urteile erfordern, die über die Modelle hinausgehen.
Danksagung: Ich danke Ralf Bergheim, Timo Henckel, Norman Isermann, Anna Klabunde, Julia Sprenger und Michael Thull für ihre sehr hilfreichen Kommentare. Auch die Anmerkungen dreier anonymer Gutachter haben sehr dazu beigetragen, den Aufsatz zu verbessern. Für alle verbliebenen Schwächen bin ich natürlich allein verantwortlich.

\section{Literatur}

Akerlof, G. A. (2007), The missing motivation in macroeconomics, American Economic Review 97(1), S. 5-36.

Allen, F., D. Gale (2009), Understanding Financial Crises, Oxford University Press.

Altunbas, Y., L. Gambacorta, D. Marques-Ibanes (2014), Does monetary policy affect bank risk? International Journal of Central Banking 10(1), S. 95-135.

Arthur, W. B. (1989), Competing, technologies, increasing returns, and lock-in by historical events, Economics Journal 99, S. $116-31$.

Arthur, W. B. (2015), Complexity economics: A different framework for economic thought. In: W. B. Arthur. Complexity and the economy, Oxford University Press, S. 1-29.

Assenza, T., D. Delli Gatti (2013), E pluribus unum: Macroeconomic modeling for multi-agent economies, Journal of Economic Dynamics and Control 37(8), S. 1659-82.

Beinhocker, E. (2007), The origin of wealth: Evolution, complexity, and the radical remaking of economics, Random House.

Blanchard, O. (2009), The state of macro, Annual Review of Econonomics 1, S. 209-28.

Brock, W. A., S. N. Durlauf (2006), Social Interactions and Macroeconomics. In: D. Colander (Hrsg.), Post-Walrasian Macroeconomics: Beyond the Dynamic Stochastic General Equilibrium Model, Cambridge University Press, S. 97-115.

Brunnermeier, M. K. (2009), Deciphering the liqidity and credit crunch 2007-08, Journal of Economic Perspectives 23(1), S. 77-100.

Buch, C. M., S. Eickmeier, E. Prieto (2014), In search for yield? Surveybased evidence on bank risk taking, Journal of Economic Dynamics and Control 43, S. 12-30.

Buiter, W. (2009), The unfortunate uselessness of most 'state of the art' academic monetary economics. Willem Buiter's mavercon blog; http://blogs.ft.com/maverecon/2009/03/the-unfortunate-uselessness-of-most-state-of-the-art-academic-monetaryeconomics/\#axzz1qVta18qg.

Cameron, L., M. Shah (2013), Risk-taking behavior in the wake of natural disasters. NBER Discussion Paper No. 19534.

Campbell, J. Y., J. H. Cochrane (1999), By force of habit: A consumption-based explanation of aggregate stock market behavior, Journal of Political Economy 107 (April), S. 205-51.

Campbell, J. Y., J. H. Cochrane (2000), Explaining the poor performance of consumption-based asset pricing models, Journal of Finance 55 (December), S. 2863-78.

Capistrán, C., A. Timmernann (2009), Disagreement and biases in inflation expectations, Journal of Money, Credit and Banking 41 (2-3), S. 365-96. 
Carroll, C. D. (2003), Macroeconomic expectations of households and professional forecasters, Quarterly Journal of Economics 118(1), S. $269-98$.

Clarke, T. (2004), Cycles of crisis and regulation: the enduring agency and stewardship problems of corporate governance, Corporate Governance - An International Review12(2), S. 153-61.

Colander, D. (2000), The complexity vision and the teaching of economics, Edward Elgar Publishing.

Colander, D., R. P. F. Holt, J. Barkley Rosser (2011), The complexity era in economics, Review of Political Economy 23(3), S. 357-69.

Colander, D., R. Kupers, (2014), Complexity and the art of public policy - Solving society's problems from the bottom up, Princeton University Press.

Croson, R., S. Gächter (2010), The science of experimental economics, Journal of Economic Behavior and Organization 73,

S. 122-131.

Deissenberg, C. S. van der Hoog, H. Dawid (2008), EURACE: A massively parallel agent-based model of the European Economy. Working Paper, HAL Id: halshs-00339756; https://halshs.archivesouvertes.fr/halshs-00339756.

Delli Gatti, D., S. Desiderio, E. Gaffeo, P. Cirillo, M. Gallegati (2011), Macroeconomics from the bottom-up, Mailand: Springer.

Dequech, D. (2007), Neoclassical, mainstream, orthodox, and heterodox economics, Journal of Post Keynesian Economics 30(2), S. 279-302.

Dosi, G., G. Fagiolo, A. Roventini (2010), Schumpeter meeting Keynes: A policy-friendly model of endogneous growth and business cycles, Journal of Economic Dynamics and Control 34(9), S. 1748-67.

Dosi, G., G. Fagiolo, M. Napoletano, A. Roventini (2013), Income distribution, credit and fiscal policies in an agent-based Keynesian model, Journal of Economic Dynamics and Control 37(8),

S. $1598-1625$.

Durlauf, S. N. (1998), What should policymakers know about economic complexity? Washington Quarterly 21(1), S. 155-65.

Elsner, W., T. Heinrich, H. Schwardt (2014), The microeconomics of complex economies - Evolutionary, institutional, neoclassical, and complex perspectives, Academic Press.

Fontana, M. (2008), The complexity approach to economics: a paradigm shift. CESMEP Working Paper.

Guiliano, P., A. Spilimbergo (im Erscheinen), Growing up in a recession. Review of Economic Studies doi: 10.1093/restud/rdt040.

Guiso, L., P. Sapienza, L. Zingales (2013), Time varying risk aversion. EIEF Working Paper 22/13.

Heathcote, J., K. Storesletten, G.L. Violante (2009), Quantitative macroeconomics with heterogeneous households. NBER working paper No. 14768.

Heise, A. (2015), Aus dem Gleichgewicht, Forschung und Lehre 5/15, S. 376-77.

Holland, J. (2006), Studying complex adaptive systems, Journal of Systems Science and Complexity 19, S. 1-8.

Hommes, C. (2011), The heterogeneous expectations hypothesis: some evidence from the lab, Journal of Economic Dynamics and Control 35(1), S. 1-24.

Kaminsky, G., R. K. Lyons, S. L. Schmukler (2004), Managers, investors, and crises: mutual fund strategies in emerging markets, Journal of International Economics 64(1), S. 113-34.

Keen, S. (1995), Finance and economic breakdown: modeling Minsky's 'financial instability hypothesis', Journal of Post Keynesian Economics 17(4), S. 607-35.
Kirman, A. (2011), Complex economics - Individual and collective rationality, Routledge.

Kraay, A., D. McKenzie (2014), Do poverty traps exist? Assessing the evidence, Journal of Economics Perspectives 28(3), S. $127-48$.

Krusell, P., A. A. Smith, Jr. (2006), Quantitative macroeconomic models with heterogeneous agents. In: R. Blundell, W. Newey, and T. Persson (eds), Advances in Economics and Econometrics: Theory and Applications, Ninth World Congress, Econometric Society Monographs, 41, Cambridge University Press, S. 298340, 2006.

Lawson, T. (2006), The nature of heterodox economics, Cambridge Journal of Economics 30, S. 483-505.

Lorenz, E. N. (1963), Deterministic Nonperiodic Flow, Journal of the Atmospheric Sciences 20(2), S. 130-41.

Lorenz, H.-W. (1987), Strange attractors in a multisector business cycle model, Journal of Economic Behavior and Organization 8, S. 397-411.

Lucas, Robert (2003), Macroeconomic priorities, American Economic Review 93(1), S. 1-14.

Luhmann, N. (1984), Soziale Systeme. Grundriß einer allgemeinen Theorie, Suhrkamp.

Ma, S.-Y. (2007), Political science at the edge of chaos? The paradigmatic implications of historical institutionalism, International Political Science Review 28(1), S. 57-78.

Maio, P. F. (2009), Intertemporal CAPM with time-varying risk aversion. Working paper http://dx.doi.org/10.2139/ssrn.889929.

Malmendier, U., S. Nagel (2011), Depression babies: do macroeconomic experiences affect risk taking? Quarterly Journal of Economics 126(1), S. 373-416.

Malmendier, U., G. Tate, J. Yan (2011), Overconfidence and early-life experiences: the effect of managerial traits on coporate financial policies, Journal of Finance 66(5), S. 1687-1733.

Mandel, A., S. Fürst, W. Lass, F. Meissner, C. Jaeger (2009), Lagom generiC: an agent-based model of growing economies. ECF Working Paper $1 / 2009$.

Mankiw, N. G., R. Reis, J. Wolfers (2003), Disagreement about inflation expectations. NBER Macroeconomics Annual 18, S. $209-48$.

May, R. M. (1976), Simple Mathematical Models with Very Complicated Dynamics, Nature 261, S. 459-67.

McDonnell, B. (2013), Dampening Financial Regulatory Cycles. Minnesota Legal Studies Research Paper No. 13-09.

Mehra, R., E. C. Prescott (1985), The Equity Premium: A Puzzle. Journal of Monetary Economics 15, S. 145-61.

Miller, J. H., S. E. Page (2007), Complex adaptive systems, Princeton University Press.

Nirei, M., T. Stamatiou, V. Sushko (2012), Stochastic herding in financial markets. Evidence from institutional investor equity portfolios. BIS working paper 371.

North, D. C. (1991), Institutions, Journal of Economics Perspectives 5(1), S. 97-112.

Rajan, R. G. (2005), Has financial development made the world riskier? Proceedings - Economic Policy Symposium - Jackson Hole, Federal Reserve Bank of Kansas City, issue Aug, pages 313-69.

Reinhart, C. M., K. S. Rogoff, M. A. Savastano (2014), Addicted to dollars, Annals of Economics and Finance 15-1, S. 1-51.

Roos, M. W. M. (2007), Die makroökonomischen Wirkungen diskretionärer Fiskalpolitik in Deutschland - Was wissen wir empirisch? Perspektiven der Wirtschaftspolitik 8(4), S. 293-308. 
Salle, I., M. Yildizoglu, M.-A. Sénégas (2013), Inflation targeting in a learning economy: An ABM perspective, Economic Modeling 34, S. 114-28.

Schmidt-Grohé, S., M. Uribe (2012), The making of a great contraction with a liquidity trap and a jobless recovery. NBER Working Paper No. 18544.

Schoar, A., L. Zuo (2014), Shaped by booms and busts: how the economy impacts CEO careers and management styles. Working paper.

Shapiro, S. C. (1992), Artificial Intelligence. In: S. C. Shapiro. Encyclopedia of Artificial Intelligence (2nd ed.), John Wiley. S. 54-57.

Simon, H. A. (1976). Rationality as process and as product of thought, American Economic Review 68(2), S. 1-16.

Smets, F., R. Wouters (2003), An estimated dynamic stochastic general equilibrium model of the euro area, Journal of the European Economic Association 1(5), S. 1123-75.

Souleles, N. S. (2004), Expectations, heterogeneous forecast errors, and consumption: micro evidence from the Michigan Consumer Sentiment Survey, Journal of Money, Credit and Banking 36(1), S. $39-72$.
The Economist (2009), What went wrong with economics? 16 July 2009, http://www.economist.com/node/14031376.

Trichet, J. C. (2012), The Malcolm Wiener Lecture in International Political Economy - 22 March 2012, Harvard University. http:// forum.iop.harvard.edu/content/malcolm-wiener-lecture-international-political-economy-delivered-jean-claude-trichet.

Van den Berg, M., R. Fort, K. Burger (2009), Natural hazards and risk aversion: Experimental evidence from Latin America. Paper provided by International Association of Agricultural Economists in its series 2009 Conference, August 16-22, 2009, Beijing, China with number 51394.

Velupillai K. V. (2005), Computability, Complexity and Constructivity in Economic Analysis, Wiley-Blackwell.

Von Bertalanffy, L. (1976), General system theory: Foundations, development, applications, Penguin University Books.

Waldrop, M. M. (1992), Complexity: The Emerging Science at the Edge of Order and Chaos, Simon \& Schuster.

Wiener, N. (1948), Cybernetics, or Control and Communication in the Animal and the Machine, MIT Press.

Wolfram, S. (2002), A new kind of science, Wolfram Media. 